(the last integral has been taken from a table ${ }^{11}$ ). Therefore, $E_{0}$ behaves like $(\ln H)^{2}$ for large values of $H$. A more careful, tedious, but straightforward study of (3), with the use of majorizations and minorizations, gives the following more precise result for the asymptotic behavior of $E_{0}$ :

$$
\begin{array}{r}
E_{0}=m c^{2}+(\alpha / 4 \pi) m c^{2}\left\{\left[\ln \left(2 e \hbar H / m^{2} c^{3}\right)\right.\right. \\
\left.\left.-C-\frac{3}{2}\right]^{2}+A+\cdots\right\},
\end{array}
$$

${ }^{11}$ I. S. Gradshteyn and I. M. Ryzhik, Table of Integrals, Series and Products, edited by A. Jeffrey (Academic Press Inc., New York, 1965). where $C=0.577$ is Euler's constant, and where $A$ is a numerical constant for which we have only found bounds : $-6<A<7$.

One readily sees from (3) that even for tremendous values of $H$ (the characteristic field $m^{2} c^{3} / e \hbar$ being $4.4 \times 10^{13} \mathrm{G}$ ), the radiative correction to $E_{0}$ remains of relative order $\alpha$. In particular, $E_{0}$ certainly does not vanish at $H=(4 \pi / \alpha)\left(m^{2} c^{3} / e \hbar\right)=7.6 \times 10^{16} \mathrm{G}$, a field value for which (1) is not valid. Some doubts about the limits of validity of the anomalous magnetic moment concept have actually been raised by the authors of Ref. 2 themselves.

\title{
Interpretation of a Unified Theory of Gravitation and Symmetry Breaking*
}

\author{
David Peak and Akira Inomata \\ Department of Physics, State University of New York, Albany, New York 12203
}

(Received 15 July 1969)

The formalism of Moen and Moffat is interpreted as a Yang-Mills theory set in a space-time generally endowed with curvature and torsion.

$I^{N}$ a recent paper, ${ }^{1}$ Moen and Moffat describe the possibility of a generalized definition of "parallel" transport of a vector nonet [an element of the tensor representation of the combined group of space-time and $U(3)$ transformations] resulting in (a) a connection between space-time and internal symmetries without reference to a "supergroup" and (b) unitary symmetry breaking induced by the presence of a zero-mass boson (to first approximation). We show that it is possible to interpret the formalism in this work as an extended Yang-Mills theory. From this point of view we see that a total symmetry group is already "embedded" in the theory, and that the character of the background space-time is sufficient to break the internal symmetry.

To see how it may be possible to make the aforementioned interpretation, we first review some aspects of a local gauge theory set in a curved background. At the outset there is, presumably, a matter field which displays a unitary symmetry characterized by ${ }^{2}$

$$
\psi^{\prime}(x)=S^{-1}(x) \psi(x) .
$$

The entities generically designated $S$ are taken to be matrix representations of elements of a group of internal transformations, and are by assumption functions of the space-time coordinates of the event point at which the transformation is made. The internal degrees of freedom of the $\psi$ field are thus adjustable at all other

\footnotetext{
* Supported in part by the National Science Foundation.

1 I. O. Moen and J. W. Moffat, Phys. Rev. 179, 1233 (1969) herein this paper shall be referred to as $\mathrm{I}$.

${ }^{2}$ C. N. Yang and R. L. Mills, Phys. Rev. 96, 191 (1954).
}

points of space-time, in keeping with the requirements of a local picture of interaction. To ensure the invariance of the dynamical structure of this system, it is necessary to introduce auxiliary field operators $B_{\mu}$ that couple universally with the various $\psi$ components, and which transform under local internal group action as

$$
B^{\prime}{ }_{\mu}=S^{-1}\left(B_{\mu} S-\nabla_{\mu} S\right) \text {. }
$$

Here $\nabla_{\mu}$ denotes the relevant space-time covariant derivative with respect to the $\mu$ th coordinate.

In a sense, the $B_{\mu}$ fields are like components of an affine connection ${ }^{3}$; as a consequence, we may define a totally covariant derivative operator expressed symbolically as

$$
D_{\mu}=\nabla_{\mu}+B_{\mu} \text {. }
$$

$D_{\mu}$ commutes with both space-time and internal transformations, and serves to establish a meaning for a parallel transport of fields with mixed indices. In terms of the vector nonets mentioned in $\mathrm{I}$, the operation of $D_{\mu}$ provides, for example,

$D_{\nu} A^{\sigma i}=\nabla_{\nu} A^{\sigma i}+B_{\nu}{ }_{j} A^{\sigma j}=\partial_{\nu} A^{\sigma i}+\left\{\begin{array}{c}\sigma \\ \mu \nu\end{array}\right\} A^{\mu i}+B_{\nu}{ }_{j} A^{\sigma j}$

where Greek indices refer to space-time structure, Latin indices to internal.

Now, the covariant derivative defined in $I$ is just such an operator, that is, it measures the effect of the total variation of fields. As expressed in that work, the

\footnotetext{
${ }^{3}$ See, e.g., J. L. Anderson, Principles of Relativity Pliysics (Academic Press Inc., New York, 1967), p. 44.
} 
covariant derivative of a contravariant vector nonet is

$$
A^{\sigma i}{ }_{\nu}=\partial_{\nu} A^{\sigma i}+h^{i}{ }_{j k} \Gamma_{\mu \nu}{ }^{\sigma j} A^{\mu k},
$$

which the $h^{i}{ }_{j k}$ given in terms of the conventional $f^{i}{ }_{j k}$ and $d^{i}{ }_{j k}$ of $U(3)$ symmetry $y^{4}$ as

$$
h^{i_{j k}}=(1-\alpha) f_{j k}+\alpha d^{i}{ }_{j k} .
$$

The right-hand side of Eq. (5) is obviously

$$
\partial_{\nu} A^{\sigma i}+h^{i}{ }_{0 k} \Gamma_{\mu \nu}{ }^{\sigma 0} A^{\mu k}+h^{i}{ }_{a k} \Gamma_{\mu \nu}{ }^{\sigma a} A_{\mu}{ }^{k},
$$

where the sum on $a$ is 1-8. Consideration of the transformation law

$$
\Gamma_{\mu \nu}^{\prime}{ }_{\mu i}^{\lambda i}=\frac{\partial x^{\prime \lambda}}{\partial x^{\alpha}} \frac{\partial x^{\beta}}{\partial x^{\prime \mu}} \frac{\partial x^{\gamma}}{\partial x^{\prime \nu}} \Gamma_{\beta \gamma}{ }^{\alpha i}+\frac{\partial^{2} x^{\prime \lambda}}{\partial x^{\alpha} \partial x^{\beta}} \frac{\partial x^{\alpha}}{\partial x^{\prime \mu}} \frac{\partial x^{\beta}}{\partial x^{\prime \nu}} \delta^{i}
$$

shows that, under change of coordinates, only the unitary scalar component of $\Gamma_{\mu \nu}{ }^{\lambda i}$ transforms as a connection while the remaining internal components transform as space-time tensors. Hence, it is plausible to interpret (5) as (4) by allowing the identifications

and

$$
\begin{gathered}
A^{\sigma i}{ }_{; \nu} \rightarrow D_{\nu} A^{\sigma i}, \\
h_{0 k}^{i} \Gamma_{\mu \nu}{ }^{\sigma 0}\left(=\delta^{i}{ }_{k} \beta \Gamma_{\mu \nu}{ }^{\sigma 0}\right) \rightarrow \delta^{i}{ }_{k}\left\{\begin{array}{c}
\sigma \\
\mu \nu
\end{array}\right\},
\end{gathered}
$$

$$
h_{a k}^{i} \Gamma_{\mu \nu}{ }^{\sigma a} \rightarrow \delta^{\sigma}{ }_{\mu} B_{\nu}{ }^{i}{ }_{k} .
$$

In fact, the second replacement is already given in I [Eq. (59)]. After interpretation, assuming as in I that internal transformations may be made pathindependent, we are always able to select an internal basis such that the third term of (7) is zero. ${ }^{5}$ Consequently, the total divergence of a vector density. nonet $\mathcal{V}^{\mu i}$,

becomes

$$
D_{\mu} \mho^{\mu i}=\partial_{\mu} \mho^{\mu i}+2\left\{\begin{array}{c}
\sigma \\
{[\mu \sigma]}
\end{array}\right\} \mho^{\mu i}+B_{\mu}{ }_{i}{ }^{\prime} \mho^{\mu j},
$$

$$
D_{\mu} \mho^{\mu i}=\partial_{\mu} \mho^{\mu i}+2\left\{\begin{array}{c}
\sigma \\
{[\mu \sigma]}
\end{array}\right\} \mho^{\mu i} \text {. }
$$

As a result, the conservation law

yields

$$
D_{\mu} \mathcal{V}^{\mu i}=0
$$

$$
\dot{F}^{i}(t)=\int \partial_{\mu} \mathcal{U}^{\mu i} d^{3} x=-2 \int\left\{\begin{array}{c}
\sigma \\
{[\mu \sigma]}
\end{array}\right\} \mho^{\mu i} d^{3} x .
$$

${ }^{4}$ M. Gell-Mann, Phys. Rev. 125, 1067 (1962).

${ }^{5}$ H. G. Loos, J. Math. Phys. 8, 2114 (1967).

The symmetry of the $F$-spin operators is broken, even in the case of zero Yang-Mills fields, by the unconventional space-time structure available in our hypotheses. The right-hand side of (10) vanishes, we note, both in the event of a torsion-free space-time and when the torsion present is completely antisymmetric.

Let us examine, in the light of our interpretation, statements (a) and (b) given initially. The assumptions in I appear tacitly to include a supergroup, namely, the direct product of space-time and internal groups. One then sees a trivial combination of the two sets of symmetries, a situation manifested in the vanishing of the Yang-Mills fields. On the other hand, symmetry breaking is still feasible as a result of the assumed torsion. The torsion acts as an independent field which couples to the current $\mathcal{V}^{\mu i}$ to break the unitary symmetry, but the unambiguous identification of a particle with this field is problematic. ${ }^{6}$

A slight modification in the unitary transformation laws given in I provides a nontrivial local gauge picture, replete with symmetry breaking even in the ordinary Minkowski background. If we let the vector nonets transform internally as

$$
\bar{\delta} A^{\sigma i}(x)=i \epsilon^{j}(x) L_{j k}^{i} A^{\sigma k}(x),
$$

the variation of $A^{\sigma i} ; \nu$ gives a "connectionlike" law for $h^{i}{ }_{j k} \Gamma_{\mu \nu}{ }^{\sigma j}$

$$
\begin{array}{r}
\bar{\delta}\left(h^{i}{ }_{j k} \Gamma_{\mu \nu}{ }^{\sigma j}\right)=i \epsilon^{n}\left(L_{n m}{ }^{i} h^{m}{ }_{j k} \Gamma_{\mu \nu}{ }^{\sigma j}-L_{n k}{ }^{m} h^{i}{ }_{j m} \Gamma_{\mu \nu}{ }^{\sigma j}\right) \\
-i\left(\partial_{\nu} \epsilon^{j}\right) L_{k j}{ }^{i} \delta^{\sigma}{ }_{\mu} .
\end{array}
$$

Since the parameters $\epsilon^{j}(x)$ are taken as scalar-valued functions of space-time, (12) is the statement in I language of the infinitesimal version of (2). With the wider generality, (8) and (9) imply

$$
\dot{F}^{i}(t)=-2 \int\left\{\begin{array}{c}
\sigma \\
{[\mu \sigma]}
\end{array}\right\} \mho^{\mu i} d^{3} x-\int B_{\mu}{ }^{i}{ }_{j} \mho^{\mu j} d^{3} x
$$

which indicates that the coupling of the Yang-Mills field to the current density alone is sufficient to break the symmetry. In the usual theory ${ }^{2}$ massless spin-1 bosons are associated with the $B_{\mu}$ fields; these can be held responsible for the breaking (13). The prototype (and as yet singular) example is, as mentioned in I, that of the electromagnetic potentials $A_{\mu}$.

\footnotetext{
${ }^{6}$ See, e.g., R. Finkelstein, J. Math. Phys. 1, 440 (1960).
} 